\title{
Design and Application of General Calculation Model for Real Interest Rate of Loan and Installment
}

\author{
Jianli Han ${ }^{1, a}$, Jianyong Bai ${ }^{1, b}$ \\ ${ }^{1}$ School of Economics and Management, Zaozhuang University, Zaozhuang 277160, China. \\ a165698650@qq.com, b157003974@qq.com
}

\begin{abstract}
Based on Excel, this paper designs a general calculation model of real interest rates for loans and instalments. The model is very applicable and can easily calculate the real interest rates of various types of loans and instalments. In addition, the pretax capital cost of bond financing and the gain on bond investments can be calculated. The design of the model is simple. The interface is simple and beautiful. The operation is easy. The model can automatically adjust the form according to the input data. The multi scheme comparison can be realized easily with the scenario manager tool.
\end{abstract}

Keywords: Real interest rate; loan; installment; scenario manager; excel.

\section{贷款和分期付款实际利率通用计算模型设计和应用 \\ 韩建丽 ${ }^{1}$, 白建勇 ${ }^{1}$ \\ 本庄学院经济与管理学院, 本庄 中国}

摘 要: 论文基于Excel设计了一个贷款和分期付款的实际利率的通用计算模型, 该模型适用 性很强，可以很方便地计算出各类贷款和分期付款的实际利率，此外还可以计算出债券融资 的税前资本成本和债券投资的收益率等。该模型设计方法简单, 界面简洁美观, 操作简便, 能够根据输入的数据自动调整表格, 结合方案管理器工具可以轻松实现多方案对比。

关键词：实际利率；贷款；分期付款；方案管理器；Excel

\section{1. 前言}

近几年来, 互联网贷款平台和小额贷款公司蓬勃发展, 各种新型贷款产品不断涌现。传统商 业银行为适应市场竞争, 也不断推出各类新的贷款产品。与此同时, 具有融资性质的各种分 期付款也让人眼花缭乱。借款者和消费者如何从纷繁复杂的各类贷款和分期付款中做出选择, 融资成本, 即融资的实际利率, 无疑是他们要重点考虑的因素。

然而, 为吸引客户, 贷款和分期付款产品宣传的利率与实际利率常常不符。例如（例1）, 某 银行推出一种贷款产品, 采用等额本息偿还, 宣传的利率是 1 年期 $3.09 \%, 2-3$ 年期 $3.27 \%$ 。如 果贷款 100000 元, 期限 3 年, 每月偿还 $(100000+100000 \times 3.27 \% \times 3) / 36=3050$ 元, 同样的方法 计算出，期限 2 年每月偿还 4440 元，期限 1 年每月偿还 8591 元。事实上，该贷款的实际利率远 高于宣传的利率。

甚至有些贷款和分期付款产品一方面宣传零利率, 一方面收取各种手续费、服务费。例如 (例 2 ）, 某信用卡账单分期, 零利率, 月服务费率 $0.6 \%$, 分 12 期, 服务费率 8 折优惠, 如果分期总 额 10000 元，则每月服务费 $10000 \times 0.6 \% \times 0.8=48$ 元，每月需还款 $10000 / 12+48=881.33$ 元。 当然, 还有一些分期付款产品根本不提及利率问题。例如（例 3 ）, 某省脐血库脐带血 20 年储 存费用, 如果一次性付清为 18580 元; 如果分三年付清, 需首付 4980 元, 以后三年每年付 5300 元, 共计 20880 元; 如果分五年付清, 需首付 3980 元, 以后五年每年付 3720 元, 共计 22580 元。 因此，为了更好地进行融资决策，需要计算各种贷款和分期付款的实际利率。 


\section{2. 贷款和分期付款实际利率的计算方法}

贷款种类繁多, 其实际利率受到还本和付息方式的影响。一般来说, 长期贷款常见的还本付 息方式有等额本息还款法、等额本金还款法、分期付息到期还本和到期一次还本付息等方式; 短期贷款常见的付息方式有收款法（贷款到期时付息）、贴现法（贷款发放时付息）和加息 法等。等额本息还款法的实际利率与名义利率近似，而加息法虽然也是每期偿还相同金额的 本息, 但其实际利率近似是名义利率的两倍。例1中的等额本息偿还, 实际上属于加息法付息, 实际利率是宣传的利率的两倍左右。一般的借款者并非专业人士, 很难区分出某个贷款产品 具体的还本付息方式，并进而算出实际利率。

根据最新的会计准则, 实际利率, 是指将金融资产或金融负债在预计存续期的估计未来现金 流量, 折现为该金融资产账面余额或该金融负债摊余成本所使用的利率。通俗地讲，实际利 率就是使现金流入现值等于现金流出现值的折现率, 也就是净现值等于零的折现率, 即内含报 酬率。要计算出贷款和分期付款的实际利率, 可以用逐步测试法和插值法, 也可以用 Exce1 中的 IRR 函数或者 RATE 函数。IRR 函数适用范围较广, 但有时较为繁琐; RATE 函数较简洁, 但主要适用于各期付款额相同的情况。

IRR 函数和 RATE 函数计算出的是每个付款期的利率。为便于比较, 实际利率通常需统一换算 成实际年利率。实际年利率 $=(1+\text { 付款期利率 })^{\wedge}$ 每年的付款期数 -1 。

为简化计算, 这里设计一个适用于各类贷款和分期付款实际利率计算的通用模型, 只需要输 入各期的收付款额等数据, 就可以直接得出实际利率。

\section{3. 贷款和分期付款实际利率计算模型设计}

本文用Exce12010软件进行贷款和分期付款实际利率计算模型设计, 模型设计效果如图1所示, 具体设计步骤如下:

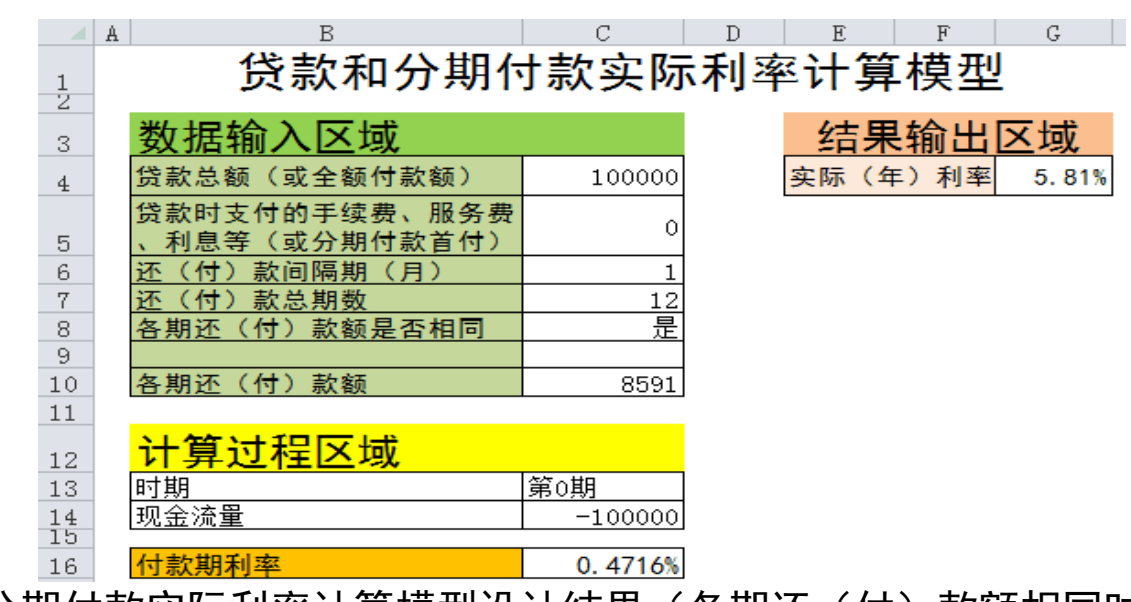

图1 贷款和分期付款实际利率计算模型设计结果（各期还（付）款额相同时的模型效果）

3.1. 数据输入区域设计

(1) 输入文本。合并 B3:C3单元格, 输入文本 “数据输入区域”。在 B4:B8单元格区域, 依 次输入文本 “贷款总额（或全额付款额）”、“贷款时支付的手续费、服务费、利息等（或分 期付款首付）”、“还（付）款间隔期（月）”、“还（付）款总期数”、“各期还（付）款 额是否相同”，在 B10单元格输入文本 “各期还（付）款额”。

(2) 设置 C8单元格。使 C8单元格只能为 “是” 或 “否”。方法: 单击【数据】选项卡下【数 据工具】中【数据有效性】下【数据有效性】命令, 弹出【数据有效性】对话框, 在其【设 置】选项卡的【有效性条件】中【允许】下拉列表中选择 “序列”，在【来源】中输入 “是, 否”。这样在 C8单元格中就产生一个下拉箭头, 只能选择 “是” 或 “否”。 
（3）设置第 9 行中的期数显示。当 C8单元格为 “是” 即各期还 (付) 款额相同时，第 9 行显示 空白（如图1）。当 C8单元格为 “否” 即各期还（付）款额不同时，可以输入各期不同的金额 （当然也可以输入相同的金额，如图2），因而模型应能够根据 “还 (付) 款总期数” 自动调 整表格，以便于使用者填写。例如，图2中所示，当 “还（付）款总期数” 为 “ 12 ”，“各期 还（付）款额是否相同” 为 “否” 时，第 9 行应自动显示出第 1 期至第 12 期。

\begin{tabular}{|c|c|c|c|c|c|c|c|c|c|c|c|c|c|c|}
\hline 7 & 还（付）款总期数 & 12 & & & & & & & & & & & & \\
\hline 8 & 各期还（付）款额是否相同 & 否 & & & & & & & & & & & & \\
\hline 9 & & 第1期 & 第2期 & 第3期 & 第4期 & 第5期 & 第6期 & 第7期 & 第8期 & 第9期 & 第10期 & 第11期 & 第12期 & \\
\hline 10 & 各期还（付）款额 & 8591 & 8591 & 8591 & 8591 & 8591 & 8591 & 8591 & 8591 & 8591 & 8591 & 8591 & 8591 & \\
\hline 11 & & & & & & & & & & & & & & \\
\hline 12 & 计算过程区域 & & & & & & & & & & & & & \\
\hline 13 & 时期 & 第0期 & 第1期 & 第2期 & 第3期 & 第4期 & 第5期 & 第6期 & 第7期 & 第8期 & 第9期 & 第10期 & 第11期 & 第12期 \\
\hline$\frac{14}{15}$ & 现金流量 & -100000 & 8591 & 8591 & 8591 & 8591 & 8591 & 8591 & 8591 & 8591 & 8591 & 8591 & 8591 & 8591 \\
\hline 16 & 付款期利率 & $0.4716 \%$ & & & & & & & & & & & & \\
\hline
\end{tabular}

图2 各期还（付）款额不同时的模型效果（部分）

设置方法如下: 首先在 $C 9$ 单元格中输入公式: $=\mathrm{IF}\left(\mathrm{C} 8={ }^{\prime \prime}\right.$ 否" $, 1,{ }^{\prime \prime}$ " $)$, 然后在 D9单元格中输入 公式: $=\operatorname{IF}(C 9<\$ C \$ 7, C 9+1, "$ " $)$, 并将该公式向右复制。由于还款期数一般不超过 50 年 (每月 1 期, 共600期）, 因此这里将公式向右复制至 WD9单元格, 这样能够实现最多600期的显示。为 显示成 “第*期” 的形式, 选中 C9: WD9单元格区域, 打开【设置单元格格式】对话框, 选择【数 字】选项下【自定义】，在【类型】中输入 “"第” 0 ”期””。

\section{2. 计算过程区域设计}

(1) 输入文本。合并 B12:C12单元格，输入文本 “计算过程区域”。在 B13，B14，B16，C13 单元格中依次输入 “时期”、“现金流量”、“付款期利率”、“第0期”。

(2) 编辑公式。在 C14 单元格中输入公式: $=-(C 4-C 5)$ 。在 D13单元格中输入公式: $=C 9$, 并 将该公式向右复制至 WE13单元格。在 D14单元格中输入公式: =IF (D13<=\$C $\left.\$ 7, C 10,{ }^{\prime \prime \prime}\right)$, 并向 右复制至WE14单元格, 该公式能保证在数据输入区域多输入的无用数据, 不会影响计算结果。 在 C16单元格中输入公式: =IF (C8="否", IRR (C14:WE14), RATE (C7, -C10, C4-C5))。

\section{3. 结果输出区域设计}

(1) 输入文本。合并 E3:G3单元格, 输入文本 “结果输出区域”。合并 E4:F4单元格, 输入 文本 “实际 (年) 利率”。

(2) 设置 G4单元格。在该单元格中输入公式: $=(1+\mathrm{C} 16) \wedge(12 / \mathrm{C} 6)-1$, 即可计算出实际年利率 数值。按照惯例, 可将该单元格设置成百分比并保留两位小数的形式。

\section{4 . 其他设置}

（1）常规设置。根据需要设置字体、字号、边框、底纹等, 并取消网格线。

（2）设置边框随期数自动调整。第 $9 、 10$ 行和第 $13 、 14$ 行的边框应能够根据期数自动调整。 以第9、10行为例, 设置方法如下: 选中 D9: WD10单元格区域, 单击【开始】选项卡下【样式】 中【条件格式】下【新建规则】命令, 打开【新建格式规则】对话框, 在【选择规则类型】中 选择 “使用公式确定要设置格式的单元格”, 在【为符合此公式的值设置格式】中输入: $=0 R(D \$ 9\langle>” \prime)$, 单击右下角【格式】按钮打开【设置单元格格式】对话框, 在【边框】中选择 “外边框”，然后确定即可。

(3) 隐藏计算过程区域。为使模型更加简洁, 可隐藏计算过程区域。方法: 选中第12至16行, 单击右键, 在弹出的快捷菜单中, 选择【隐藏】命令。 


\section{4. 贷款和分期付款实际利率计算模型的应用}

\section{1 . 基本应用}

基本应用，是指利用该模型可方便地进行各类贷款和分期付款实际利率的计算。 如例 1 中的贷款。贷款金额 100000 元，贷款时无其他费用，1年期，每月偿还8591元，即还款 间隔期为 1 月, 还款总期数为 12 期, 各期还款额相同, 为8591元。因此, 只需在 C4:C8单元格 区域和 $\mathrm{C} 10$ 单元格中，依次输入 $100000 、 0 、 1 、 12$ 、是、8591，即可得到 1 年期贷款的实际年 利率是 $5.81 \%$ （如图1所示）。同样的道理，可得到2-3年期贷款的实际年利率是 $6.35 \%$ 。它们 均明显高于宣传的利率1年期 $3.09 \% ， 2-3$ 年期 $3.27 \%$ 。

例 2 中的信用卡账单分期。分期总额 10000 元, 每月还款 881.33 元。在 C4:C8单元格区域和 C10 单元格中，依次输入 $10000 、 0 、 1 、 12$ 、是、881.33，可得到该分期的实际年利率是 $10.98 \%$ 。 如果认为该分期的年利率是 $0.6 \% \times 0.8 \times 12=5.76 \%$ ，则明显低估了该项融资的成本。 例 3 中的分期付款。在 $\mathrm{C} 4$ : C8单元格区域和 $\mathrm{C} 10$ 单元格中，依次输入 $18580 、 4980 、 12 、 3 、$ 是、 5300 , 可得到分三年付清的实际年利率是 $8.24 \%$ 。同样的, 依次输入 $18580 、 3980 、 12 、 5 、$ 是、 3720 , 得到分五年付清的实际年利率是 $8.65 \%$ 。如果消费者能够以小于 $8.24 \%$ 的成本融到资金, 或者投资收益小于 $8.24 \%$, 可以选择一次性付清。

\section{2. 其他应用}

\section{2. 1 债券融资税前资本成本的计算}

例 4 ABC 公司拟发行 30 年期的债券, 面值 1000 元，利率 $10 \%$ (按年付息)，所得税税率 40\%, 发 行费用率为面值的 $1 \%$ 。

经过分析，在 C4:C8单元格区域依次输入 1000、10、12、30、否，每期利息是 $1000 \times 10 \%=100$ 元, 所以第 $1-29$ 期的付款金额输入 100 , 第30期的金额输入 1100 。该模型能自动算出该债券融 资的税前资本成本是 $10.11 \%$ 。

\subsection{2债券投资收益率的计算}

例 $5 \mathrm{ABC}$ 公司 $20 \times 1$ 年 2 月 1 日以 1105 元的价格购买一张面额为 1000 元的债券, 其票面利率为 $8 \%$, 每年2月1日计算并支付一次利息, 并于5年后的1月31日到期。该公司持有该债券至到期日, 计算其到期收益率。

经过分析, 在 C4:C8单元格区域依次输入 1105、0、12、5、否，第 1-4期的付款金额输入80, 第5期的金额输入 $1080 ，$ 该模型自动算出该债券投资的收益率是 $5.54 \%$ 。

\section{3. 多方案比较}

借助于方案管理器工具, 本模型能轻松实现多个方案的对比。下面仍以例 1 说明操作步骤。

1. 打开方案管理器。单击【数据】选项卡下【数据工具】中【模拟运算】下【方案管理器】命 令, 打开【方案管理器】对话框。

2. 添加方案。单击右上角【添加】按钮, 弹出【添加方案】对话框, 在【方案名】中输入 “一 年期”, 【可变单元格】中输入 $\$ C \$ 7, \$ C \$ 10$, 单击【确定】, 在打开的【方案变量值】对话框 中依次输入 $12 、 8591$, 然后单击【确定】, 返回【方案管理器】对话框。用同样的方法添加方 案二、方案三。

3. 显示比较结果。在【方案管理器】对话框中, 单击【摘要】按钮, 打开【方案摘要】对话 框, 在【结果单元格】中选择 $\$ G \$ 4$ 单元格, 单击【确定】, Exce1 会自动生成一张名为 “方案 摘要” 的工作表 (如图3所示)。为使显示结果易于理解, 已事先给相关单元格定义了名字。 


\begin{tabular}{|c|c|c|c|c|}
\hline 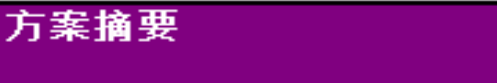 & 当前值： & 一年期 & 二年期 & 三年期 \\
\hline \multicolumn{5}{|l|}{ 可变单元格： } \\
\hline 还_付_款总期数 & 12 & 12 & 24 & 36 \\
\hline 笲期还１付_款额 & 8591 & 8591 & 4440 & 3050 \\
\hline \multicolumn{5}{|l|}{ 结果单元格： } \\
\hline 实际 年 利率 & $5.81 \%$ & $5.81 \%$ & $6.35 \%$ & $6.35 \%$ \\
\hline
\end{tabular}

图3 利用方案管理器进行多方案对比

\section{5. 总结}

贷款和分期付款的实际利率，是借款者和消费者决策时需重点考虑的因素。本文基于 Exce1 设计了一个贷款和分期付款实际利率的通用计算模型, 该模型适用性很强, 可以很方便地计 算出各类贷款和分期付款的实际利率, 此外还可以计算出债券融资的税前资本成本和债券投 资的收益率等。该模型界面简洁美观, 能够根据输入的原始数据自动调整表格, 操作简便, 结合方案管理器工具可以轻松实现多方案对比，设计方法也比较简单。

\section{References}

[1]. Fang Gobi, Wan Renin. Analysis of the calculation of nominal interest rate and real interest rate [J]. China Economist. 2010 (6).p.44-45.

[2]. Wang Chunkier. Analyze the real interest rate from the perspective of compound interest [J]. Friends of Accounting. 2014 (7) .p. 117-119. 